\title{
The role of various transporters in the placental uptake of ofloxacin in an in vitro model of human villous trophoblasts
}

This article was published in the following Dove Press journal: Drug Design, Development and Therapy

\author{
Hana Polachek ${ }^{1, *}$ \\ Nir Debotton ${ }^{2, *}$ \\ Valeria Feinshtein' \\ Mazal Rubin' \\ Zvi Ben-Zvi ${ }^{1, \neq}$ \\ Gershon Holcberg ${ }^{3}$ \\ Riad Agbaria' \\ Arik Dahan'
}

'Department of Clinical Pharmacology, School of Pharmacy, Faculty of Health

Sciences, Ben-Gurion University

of the Negev, Beer-Sheva, Israel;

${ }^{2}$ Department of Chemical Engineering,

Shenkar College of Engineering and

Design, Ramat-Gan, Israel; '3ivision

of Obstetrics and Gynecology, Soroka

University Medical Center, Faculty

of Health Sciences, Ben-Gurion

University of the Negev, Beer Sheva,

Israel

*These authors contributed equally to this work

‡Professor Zvi Ben-Zvi passed away on August 4, 2017
Correspondence: Arik Dahan Department of Clinical Pharmacology, School of Pharmacy, Faculty of Health Sciences, Ben-Gurion University of the Negev, PO Box 653, Beer-Sheva 8410501 , Israel

Tel +97286479483

Fax+97286479303

Email arikd@bgu.ac.il
Introduction: Six years after the US Food and Drug Administration approval of the broadspectrum antibiotic ofloxacin (OFLX), the chiral switching of this racemic mixture resulted in a drug composed of the L-optical isomer levofloxacin (LVFX). Since both fluoroquinolones (FQs) were introduced to the pharmaceutical market, they have been widely prescribed by physicians, with careful administration during pregnancy and breastfeeding. Therefore, the role of the influx and efflux placental transporters in the concentrations of these drugs that permeate through human placental barrier model was investigated in this study.

Methods: The contribution of major carriers on the transplacental flux of OFLX and LVFX uptake into choriocarcinoma BeWo cells was evaluated in the presence vs absence of wellknown inhibitors.

Results: Our results reveal that neither the influx transporters such as organic cation transporters, organic anion transporters, and monocarboxylate transporters nor the efflux transporters such as P-glycoprotein or breast cancer resistance protein significantly affected the transport of OFLX. In contrast, multiple transporters revealed pronounced involvement in the transfer of the levorotatory enantiomer in and out of the in vitro placental barrier. These data suggest a non-carrier-mediated mechanism of transport of the racemic mixture, while LVFX is subjected to major influx and efflux passage through the placental brush border membranes.

Conclusion: This study provides underlying insights to elucidate the governing factors that influence the flux of these FQs through organ barriers, in view of the controversial safety profile of these drugs in pregnant population.

Keywords: drug transport, fluoroquinolones, levofloxacin, ofloxacin, placenta, pregnancy

\section{Introduction}

Fluoroquinolones (FQs) are synthetic broad-spectrum antibiotic medications that are active against both Gram-positive and Gram-negative bacteria. The insertion of a 6-fluoro group into the structure of the quinolone skeleton resulted in numerous chemical entities that have been patented as potential antimicrobial agents. The fluorinated quinolones that have been approved for clinical use revealed considerably improved bactericidal potency and reduced adverse effects. ${ }^{1}$ Early generation of FQs includes norfloxacin, ciprofloxacin, and ofloxacin (OFLX), while newer generations include moxifloxacin, delafloxacin, and levofloxacin (LVFX). The antimicrobial strength of LVFX against Gram-positive and Gram-negative bacteria is superior to that of OFLX, probably because LVFX is a purified biologically active optical isomer, whereas OFLX is a racemic mixture that consists of equal amount of D- and L- enantiomers. ${ }^{2}$ At physiological $\mathrm{pH}$, both OFLX and LVFX exist as zwitterionic moieties (negative and positive ionized groups exhibiting a molecule 
with zero electrical charge), ${ }^{3}$ as illustrated in Scheme 1. Delafloxacin was most recently approved for the treatment of acute bacterial skin and skin structure infections. Delafloxacin is not a zwitterion, and it is unionized at acidic environments. Consequently, these chemical modifications allow the improved potency of the new FQ derivative. ${ }^{4}$

The majority of the FQs targets the bacterial DNA gyrase and/or topoisomerase II and IV enzymes. ${ }^{5}$ The clinical uses of these bactericidal agents are vast and include urinary, respiratory, and gastrointestinal tract infections; bone and joint infections; skin and soft tissue infections; sexually transmitted diseases; and prostatitis., ${ }^{2,6}$ While the frequent adverse effects documented for FQs include gastric, skin, and central nervous system manifestations, some of the most extensively utilized agents such as ciprofloxacin, OFLX, and LVFX are well tolerated. ${ }^{1}$ Early studies revealed that norfloxacin, ciprofloxacin, and OFLX have been found to induce structural defects in the progenies of pregnant animal models. Therefore, the clinical use of FQ is generally avoided in children and pregnant women. ${ }^{6,7}$ In fact, the US Food and Drug Administration pregnancy category rating for FQs is $\mathrm{C}$, and they should be prescribed by physicians in pregnancy only in case there is no other alternative. However, recent publications question the risks of FQs orally administered to expecting women compared to that of animal models. ${ }^{8}$ Indeed, a case study revealed no teratogenic or toxic properties for OFLX when a 36-yearold pregnant woman (during the 19th week of gestation) was treated with $200 \mathrm{mg}$ FQ bid over 6 days. ${ }^{9}$

During the growth of the human placenta, the fusion of differentiated cytotrophoblast forms the syncytiotrophoblast<smiles>CC1COc2c(N3CCN(C)CC3)c(F)cc3c(=O)c(C(=O)O)cn1c23</smiles>

\section{$\stackrel{\text { Physiological } \mathrm{pH}}{\longrightarrow}$}<smiles>C=C(O)c1cn2c3c(c(N4CCN(C)CC4)c(F)cc13)OCC2C</smiles><smiles>C[C@H]1COc2c(N3CCN(C)CC3)c(F)cc3c(=O)c(C(=O)O)cn1c23</smiles>

Physiological $\mathrm{pH}$<smiles>C[C@H]1COc2c(N3CCN(C)CC3)c(F)cc3c(=O)c(C(=O)O)cn1c23</smiles>

Scheme I Molecular structures of OFLX and LVFX and their ionization to zwitterionic moieties at physiological pH. Abbreviations: LVFX, levofloxacin; OFLX, ofloxacin. 
that completely envelops the developing placenta. ${ }^{10}$ This outmost layer of syncytium forms an effective barrier that governs the gas exchange and the transfer of nutrients or drugs. ${ }^{11}$ The ex vivo methods to investigate the function of the syncytiotrophoblast include culture of placental explants or the use of placental lobe perfusion systems. On the other hand, the in vitro approaches include isolation of cytotrophoblast populations from a whole placenta or from human pluripotent stem cells, wherein a type of syncytium is formed holding certain biological properties of the syncytiotrophoblast. Another in vitro model utilizes choriocarcinoma cultures that differentiate into multinuclear cells in the presence of certain agents such as forskolin. ${ }^{12}$ The human placental choriocarcinoma BeWo cells are commonly used as a placental barrier model exhibiting physiological feature the villous trophoblast. ${ }^{13}$ The human BeWo cell line provides an efficient technique that is well correlated with the ex vivo placental perfusion model to evaluate the asymmetric transcellular transport of various compounds. ${ }^{14,15}$ Some of these compounds include psoralen, ${ }^{16}$ bilirubin,,${ }^{17}$ toltrazuril, ${ }^{18}$ iron, ${ }^{19}$ and valproic acid. ${ }^{20,21}$

The functionality of the placental barrier protects the fetus by controlling the transport of xenobiotics from the maternal to the fetal blood circulation, mediated by passive diffusion or active transport. In general, passive diffusion is the major route of transport of lipophilic agents across the placenta. Active transport by means of highly specific transplacental transporters predominates the passage of hydrophilic molecules. This active transport across the placenta is a saturable process. ${ }^{16,17}$ The efflux transporters such as $\mathrm{P}$-glycoprotein (P-gp) and breast cancer resistance protein (BCRP) present at the apical side of the syncytiotrophoblast among other tissues. P-gp (multidrug resistance protein 1 [MDR1]) and BCRP discard their substrates apically from the syncytiotrophoblast. In contrast, the organic anion transporters (OATs), localized in the basolateral membrane, introduce their substrates into the syncytiotrophoblast and the cytotrophoblast. ${ }^{22,23}$ Although influx transport may occur also by the organic cation transporters (OCTs) and the monocarboxylate transporters (MCTs) present at the basolateral side of the human placenta, ${ }^{22}$ some of their subtypes promote a bidirectional transport of molecules into and from the syncytiotrophoblast. ${ }^{23,24}$

In view of our previous work on $\mathrm{LVFX}^{25}$ the aim of the present research was to determine and characterize the carrier-mediated placental transfer of OFLX. We sought to identify the contribution of major transporters on the transplacental flux of the drug; specifically, the comparison between the transport characteristics of the active stereoisomer (ie, LVFX) and the racemic mixture was of major focus.

\section{Materials and methods Materials}

Cimetidine was obtained from GlaxoSmithKline Consumer Healthcare (Middlesex, UK). LVFX was acquired from Hoechst Marion Roussel, Inc. (Kansas City, MO, USA), and OFLX was purchased from Aventis Pharma (Frankfurt, Germany). Omeprazole (OMZ) was obtained from AstraZeneca (Södertälje, Sweden). Calcium chloride, cyclosporine A, EDTA, Hank's Balanced Salt Solution, lactic acid, magnesium sulfate, PBS tablets, potassium chloride, potassium dihydrogen phosphate, probenecid, quercetin, sodium chloride, and verapamil were obtained from Sigma-Aldrich Co. (St Louis, MO, USA). HPLC grade acetonitrile, dichloromethane, double distilled water (DDW), hexane, and methanol were purchased from EMD Millipore (Billerica, MA, USA).

\section{Cell cultures}

The choriocarcinoma BeWo cell line (obtained from Dr B. Ugele, Ludwig-Maximilians University, Munich, Germany and was approved for use by the Ben-Gurion University Committee for ethical experimentation, No 0080-14-SOR) was cultured at $37^{\circ} \mathrm{C}$ under $5 \% \mathrm{CO}_{2}$, using DMEM/F12 enriched with 10\% heat-inactivated FBS, $2 \mathrm{mM}$ L-glutamine, $100 \mathrm{IU} / \mathrm{mL}$ penicillin, and $100 \mathrm{ng} / \mathrm{mL}$ streptomycin. All cell culture products were purchased from Biological Industries (Beit Haemek, Israel).

\section{Cellular uptake studies of OFLX and LVFX into BeWo cultures}

The cells were grown to confluent monolayers over 3-4 days in six-well plates at a concentration of approximately $2 \times 10^{6}$ cells per well. The media from each well were discarded, and the cells were washed three times with $2 \mathrm{~mL} P B S$ at $37^{\circ} \mathrm{C}$. Then, the cultures were pre-incubated with the uptake buffer (Hanks' balanced salt solution) in a shaking water bath at $37^{\circ} \mathrm{C}$.

The pre-incubation period of the time- and concentrationdependent cellular uptake experiments was 10 minutes. BeWo cells were pre-incubated over 30 minutes with the various substrates and inhibitors dissolved in the uptake buffer. The cell internalization was stopped by washing the cells with ice-cold PBS. Then, the cultures were harvested using $1 \mathrm{~mL}$ trypsin-EDTA solution. The cellular suspension was centrifuged for 15 minutes at $1,200 \times g$, and the supernatant was removed. About 250-500 $\mu \mathrm{L}$ of sterile PBS was added to the pellet that was vortexed vigorously. Consecutively, the cells 
were incubated at $-20^{\circ} \mathrm{C}$, thawed, and sonicated to obtain cell lysate containing OFLX and LVFX. The total cellular protein concentration was determined by the Lowry assay using microplate spectrophotometer at $650 \mathrm{~nm} .{ }^{26}$

\section{Quantitation of OFLX and LVFX}

The concentrations of the FQs were analyzed utilizing Hitachi HPLC system. Separation of OFLX and LVFX was carried out using Hypersil ${ }^{\mathrm{TM}}$ BDS C18 $100 \times 4.6 \mathrm{~mm}$ column, and identification of the drugs was performed using a fluorescence detector at $278_{\mathrm{EX}} / 456_{\mathrm{EM}} \mathrm{nm}$. Calibration curves ranged from 0 to $250 \mathrm{ng} / \mathrm{mL}$, while the internal standard of OFLX was LVFX and vice versa. Purification of the samples from undesired proteins was carried out with $50 \%$ acetonitrile followed by centrifugation. For lipid extraction, dichloromethanehexane (1:1) mixture was added, and the samples were vortexed vigorously and centrifuged again. Then, $20 \mu \mathrm{L}$ of the lower aqueous phase was carefully sampled and injected into the HPLC system.

\section{Statistical analyses}

Treatments replicates were subjected to statistical analyses carried out using Student's $t$-test. Differences were accepted as significant for $P<0.05$.

\section{Results}

\section{Time-dependent uptake of OFLX} compared to LVFX using

\section{choriocarcinoma cell culture}

The results presented in Figure 1 indicate that OFLX and LVFX reached a maximal uptake after 5 and 10 minutes, respectively. Following the relatively rapid maximal uptake points, LVFX maintained a rather constant concentration in the cells, while the accumulation of OFLX considerably decreased by $23.7 \%-31.6 \%$ over the remaining time intervals.

\section{Concentration-dependent uptake of OFLX compared to LVFX using a placental cell culture model}

The results in Figure 2 indicate that the uptake of LVFX into BeWO cells demonstrated two courses of actions: initial and relatively rapid uptake rates, followed by a decreased and linear rate. The internalization rate of LVFX into the cells was altered at $10 \mu \mathrm{M}$, which may propose the existence of two different mechanisms of cellular internalization. In contrast, the time-dependent cellular internalization of OFLX revealed a linear correlation $\left(R^{2}=0.96\right)$, lacking the two-phase mode of internalization observed with LVFX (Figure 2). Furthermore,

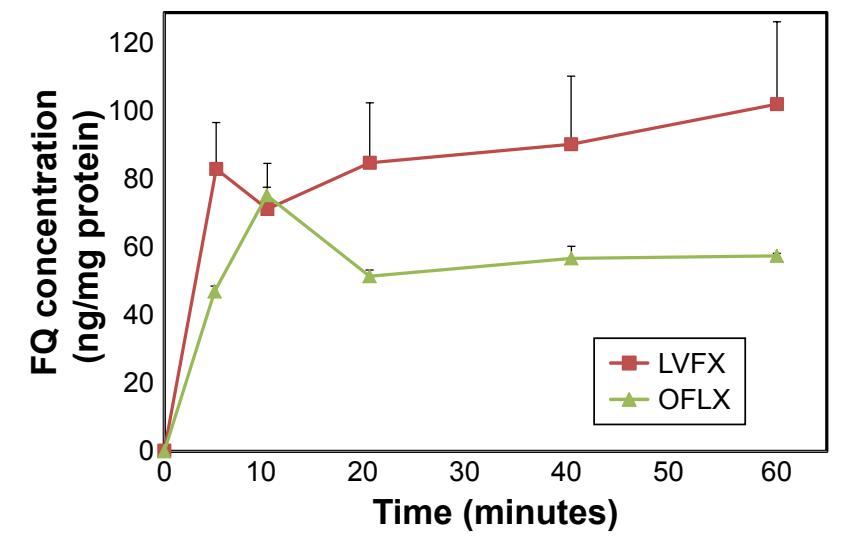

Figure I Time-dependent uptake of LVFX and OFLX.

Notes: The accumulation of the FQs is expressed as ng of drug per mg of cell protein, following incubation with BeWo cells over time. Results are presented as average $\pm S D ; n=9$.

Abbreviations: FQ, fluoroquinolone; LVFX, levofloxacin; OFLX, ofloxacin.

the uptake of OFLX exhibited this linear relationship up to a high concentration of $0.5 \mathrm{mM}\left(R^{2}=0.996\right)$, as depicted in Figure 2 (inner graph). These results may indicate a single mechanism of cellular internalization of OFLX into BeWo choriocarcinoma cells.

\section{Contribution of $\mathrm{P}$-gp inhibitors on the cellular uptake of OFLX}

The basal level of P-gp in the placental membranes of the BeWo cells was quantified earlier by our group to verify the presence of the transporter using Western blotting. ${ }^{27}$ In this study, the P-gp inhibitors, verapamil, cyclosporine A, and quercetin, were evaluated for their potential to elicit the concentration of OFLX that permeated the cells (Figure 3). The results depicted in Figure $3 \mathrm{~A}-\mathrm{C}$ present a slightly elevated cellular internalization of the FQ. Nevertheless, no statistical difference in the increase of OFLX levels was detected when the cells were incubated with the familiar P-gp inhibitor verapamil (Figure 3A). In addition, cyclosporine $\mathrm{A}$ and quercetin did not exhibit marked inhibition effect on the uptake of OFLX into the BeWo cells (Figure 3B and C, respectively). These findings indicate poor contribution of P-gp transporters to the apical efflux of OFLX from this choriocarcinoma cell model.

\section{In vitro transport of OFLX vs LVFX on BCRP inhibition in BeWo cells}

Our group previously validated the placental BCRP expression in BeWo cells, utilizing Western blotting technique. ${ }^{27}$ The present study investigated the contribution of BCRP on the efflux of OFLX and LVFX from BeWo cells, utilizing the known BCRP inhibitor OMZ. The results 


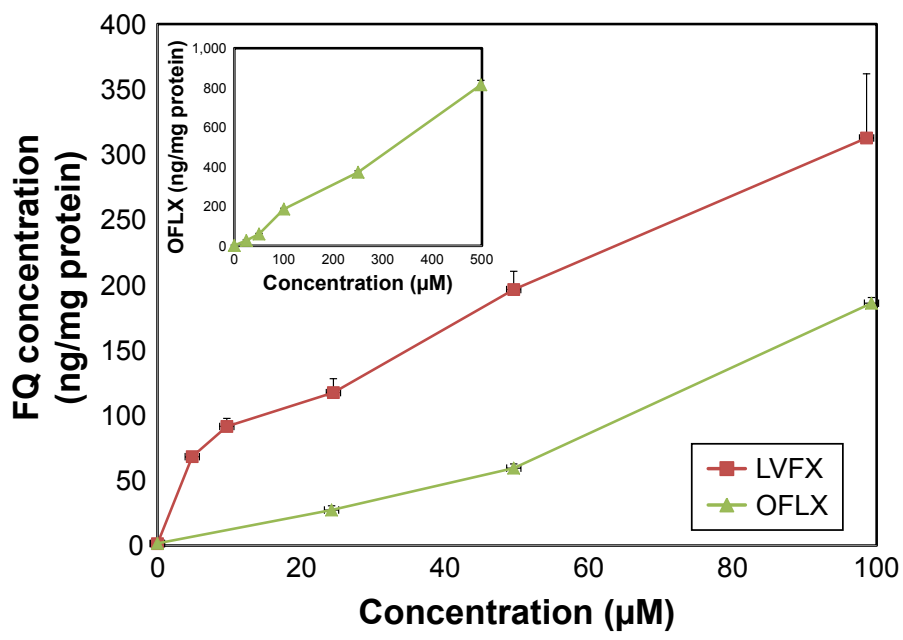

Figure 2 Concentration-dependent uptake of OFLX and LVFX.

Notes: The accumulation of the FQs is expressed as ng of drug per mg of cell protein, following incubation with BeWo cell at increasing drug concentrations. Results are presented as average $\pm S D ; n=9$.

Abbreviations: FQ, fluoroquinolone; LVFX, levofloxacin; OFLX, ofloxacin.

exhibited in Figure 4 clearly indicate that BCRP inhibition using $\mathrm{OMZ}$ showed a lack of effect in the cellular uptake of OFLX (Figure 4A). In contrast to the results obtained with OFLX, when LVFX was evaluated, OMZ considerably elevated drug levels in the cells as presented in Figure 4B $(P<0.05)$. In case of LVFX, the concentration of the drug following BCRP inhibition was 1.53-fold higher than the concentration of LVFX without any transporter inhibition.

\section{Cellular uptake of OFLX compared to LVFX following inhibition of OATs and OCTs}

OATs and OCTs, members of the SLC22A drug transporter superfamily, carry relatively small, hydrophilic organic anions and cations, respectively. ${ }^{24}$ While OATs are generally influx transporters of their ligands, some subtypes of OCTs allow influx and efflux transport of their substrates. ${ }^{23}$ In this study, the effect of the $\mathrm{H} 2$ blocker cimetidine as an OCT inhibitor, and the influence of probenecid that is known as a prominent inhibitor of OAT, were evaluated on the cellular uptake of the FQs (Figures 5 and 6, respectively).

The uptake of OFLX in BeWo cells was approximately $30 \%$ higher following incubation with cimetidine compared to the uptake of this FQ in the non-treated cultures (Figure 5); however, statistical analyses of these results reveal that this increase was not significant. Furthermore, a weak involvement of OATs was demonstrated when the inhibitor probenecid was evaluated, and as a result, the influx
A

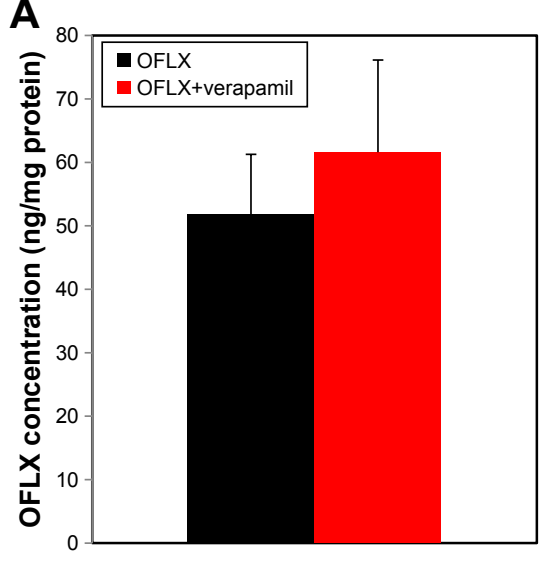

B

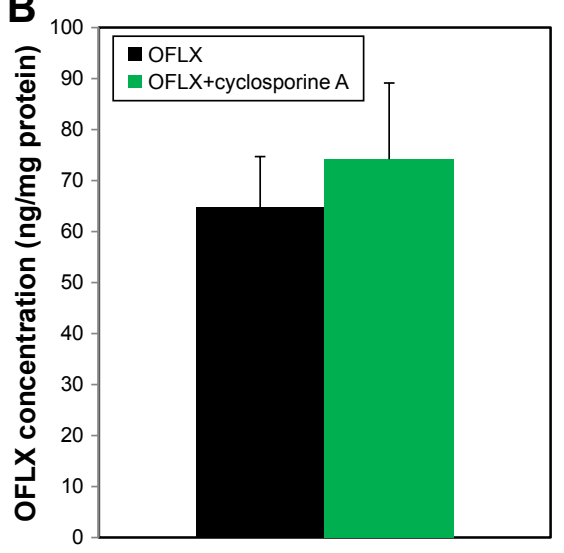

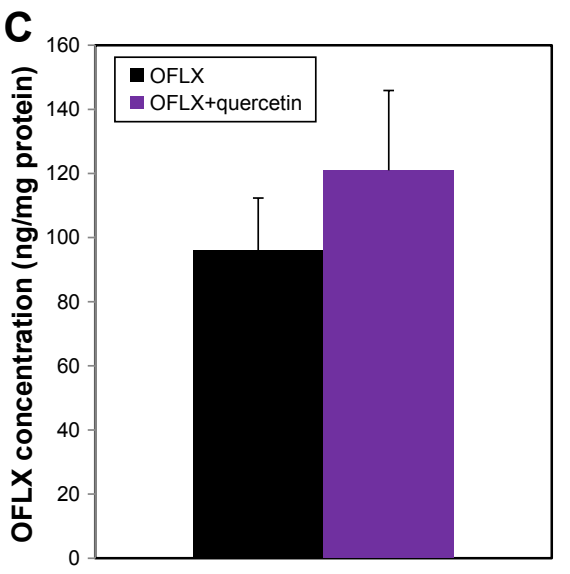

Figure 3 Influence of P-gp inhibitors on OFLX transport into BeWo cells.

Notes: (A) Treatment with $100 \mu \mathrm{M}$ of verapamil. (B) Treatment with $20 \mu \mathrm{M}$ of cyclosporine A. (C) Treatment with $10 \mu \mathrm{M}$ of quercetin. Results are presented as average $\pm S D ; n=9$.

Abbreviations: OFLX, ofloxacin; P-gP, P-glycoprotein. 
A

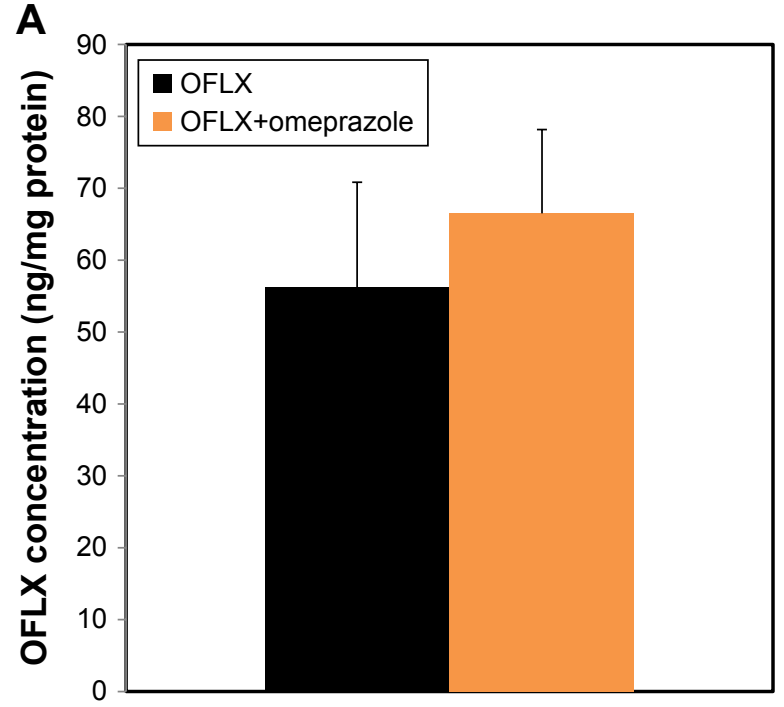

B

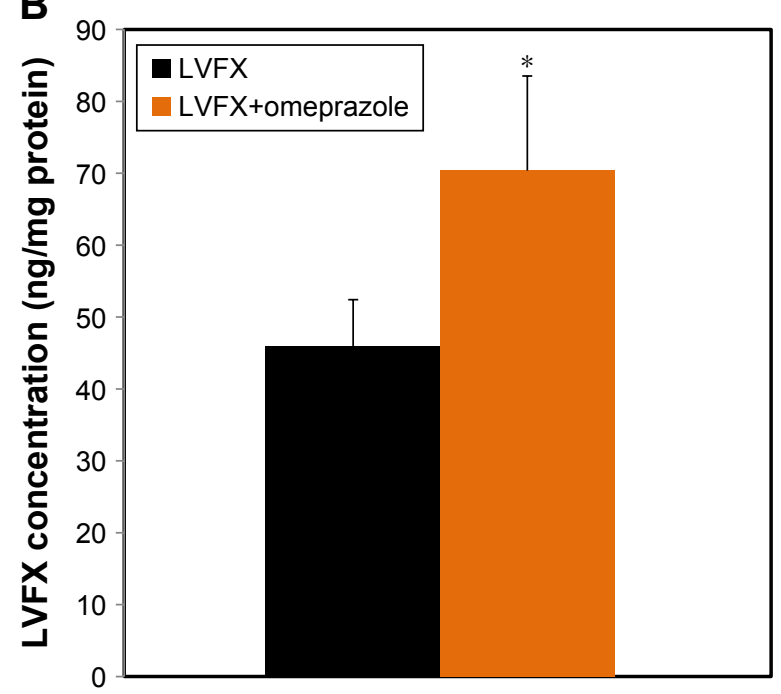

Figure 4 Effect of BCRP inhibition on the accumulation of OFLX (A) vs LVFX (B) in BeWo cells following incubation with I00 $\mu M$ of OMZ.

Notes: Results are presented as average \pm SD; $n=9 ; * P<0.05$.

Abbreviations: BCRP, breast cancer resistance protein; LVFX, levofloxacin; OFLX, ofloxacin; OMZ, omeprazole.

of OFLX into the in vitro model of human trophoblasts did not considerably decrease. Thus, although this drug is a zwitterion at physiological environment, neither OCT nor OAT displays any significant impact on OFLX cellular uptake (Figures 5 and 6A).

In contrast to OFLX, investigation of the biologically active enantiomer demonstrates that LVFX levels were significantly elevated after treatment with probenecid (Figure 6B; $P<0.05$ ). In case of LVFX, intracellular drug concentrations dropped by $\sim 35 \%$ following OATs inhibition, compared to non-treated cells.

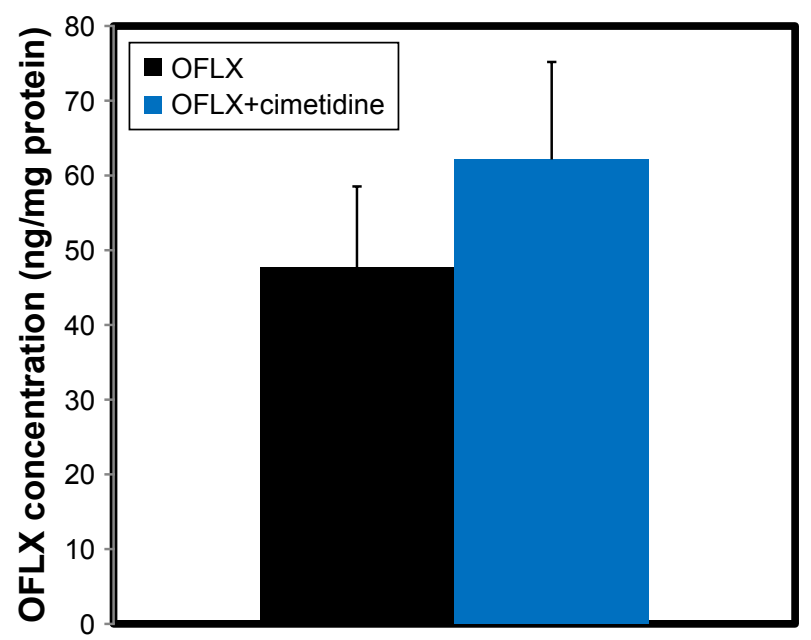

Figure 5 Contribution of OCT inhibition on the transport of OFLX into BeWo cells following treatment with $10 \mu \mathrm{M}$ cimetidine.

Notes: Results are presented as average $\pm S D ; n=9$.

Abbreviations: OCT, organic cation transporter; OFLX, ofloxacin.

\section{Effect of MCTs substrate on the in vitro transport of OFLX}

The proton-dependent MCTs in human syncytiotrophoblast generate bifunctional activity toward their substrates; therefore, monocarboxylic acid drugs may undergo influx and efflux transport across the basolateral side of the placental membrane. The results presented in Figure 7 demonstrate the influence of the MCT substrate lactic acid on the uptake of OFLX into BeWo cells. The results revealed the lack of statistically significant effect, although this typical FQ antibiotic contains a monocarboxylic acid functional group.

\section{The transporter-mediated permeation of OFLX compared to LVFX}

To summarize the contribution of the various transporters on the uptake of OFLX into BeWo choriocarcinoma cells, we calculated the percent of the uptake change of the intracellular drug measured with inhibitors vs without inhibitors. Table 1 details the results calculated from the data presented in Figures 3-7 and from the data in our earlier LVFX publication. ${ }^{25}$ It can be seen that the entire set of the tested inhibitors resulted in a marked change in the permeation of LVFX $(P<0.05)$. Nevertheless, the uptake of OFLX was only slightly modified, with no statistical significance, following treatment with identical inhibitors. The permeation of OFLX and LVFX exhibited an opposite trend with MCT substrate, as OFLX uptake change decreased and LVFX uptake change profoundly increased (Table 1). Moreover, while OMZ 
A

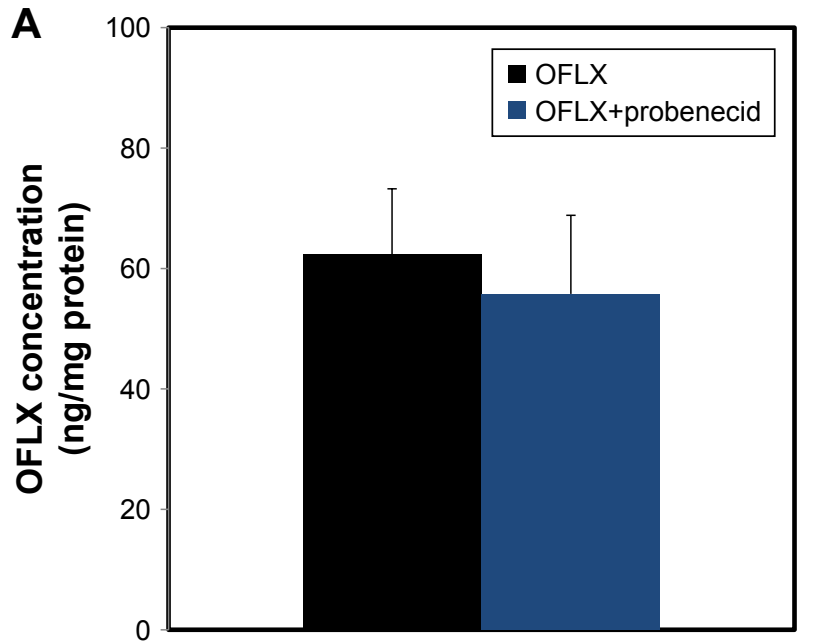

B

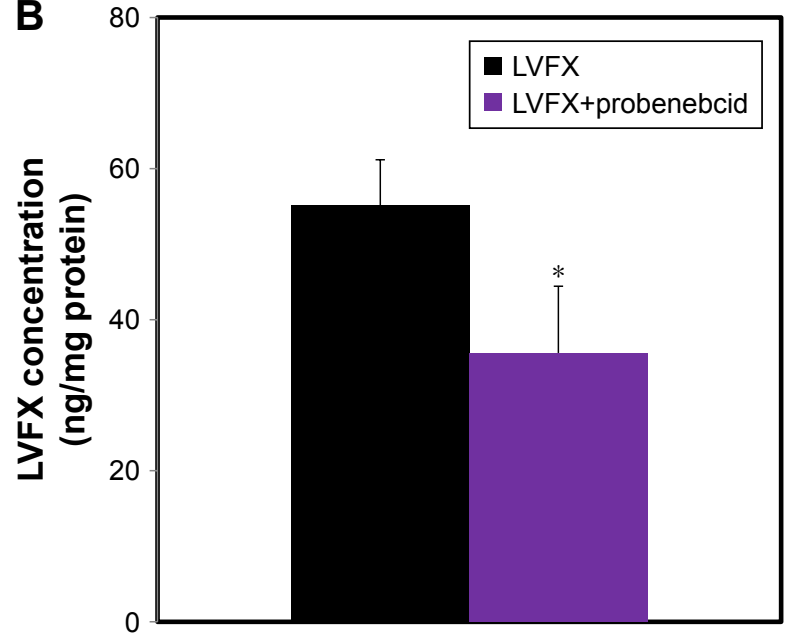

Figure 6 Accumulation of OFLX (A) vs LVFX (B) within BeWo cell line after incubation with I $\mu$ M of the OAT inhibitor probenecid. Notes: Results are presented as average $\pm S D ; n=9 ; * P<0.05$.

Abbreviations: LVFX, levofloxacin; OAT, organic anion transporter; OFLX, ofloxacin.

(BCRP inhibitor) increased and probenecid (OAT inhibitor) decreased the uptake change of LVFX, these agents did not markedly influence the OFLX cellular uptake.

\section{Discussion}

In this study, several common placental transporters were evaluated to clarify their role in the cellular transport of the antibiotic OFLX in and out of human villous trophoblasts in vitro model compared to the additional frequently prescribed FQ, LVFX.

P-gp inhibitors did not cause significant decrease in the efflux of OFLX (Figure 3), while the efflux of its levorotatory

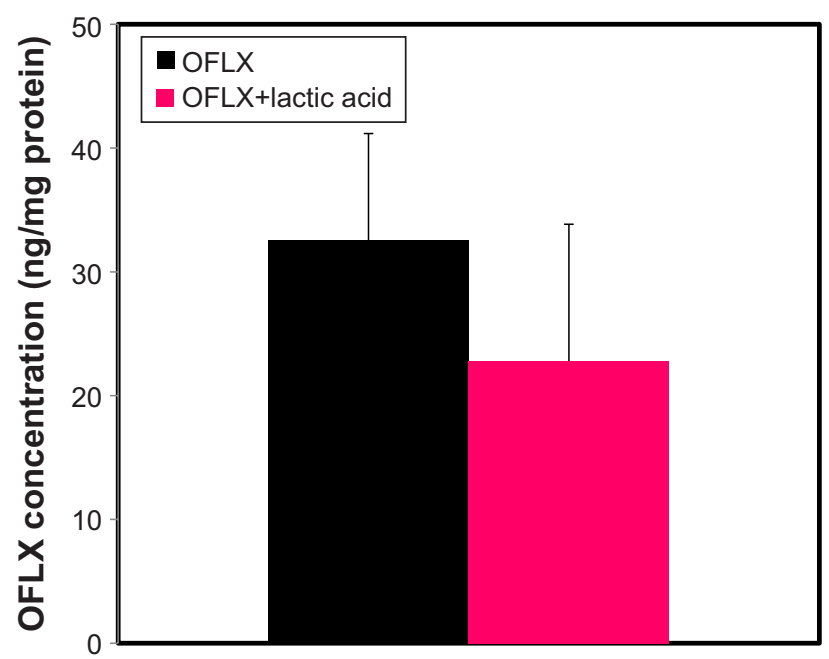

Figure 7 Influence of MCTs substrate on OFLX accumulation within BeWo cells following incubation with $5 \mu \mathrm{M}$ of lactic acid.

Notes: Results are presented as average $\pm S D ; n=9$.

Abbreviations: MCT, monocarboxylate transporter; OFLX, ofloxacin. enantiomer (LVFX) profoundly reduced in the presence of verapamil, cyclosporine A, and quercetin (Table 1). In contrast to the racemic mixture of OFLX evaluated in the presence of BCRP inhibition, the efflux of LVFX was considerably increased by OMZ, as shown in Figure 4. These findings indicate that P-gp and BCRP are not involved in the transport of the dextrorotatory enantiomer to the human placental cell cultures.

Similar results were obtained for OCT, as its inhibition by cimetidine led to non-significant effect on OFLX transport (Figure 5). It should be noted that in our previous experiments, the uptake of LVFX was markedly augmented in the presence of this inhibitor. ${ }^{25}$ In the study by Polachek et al, cimetidine was proposed as an OAT3/OCT inhibitor that increases the cellular LVFX uptake by $50 \%$, as shown

Table I Uptake change (\%) of FQs into BeWo cell cultures in the presence vs absence of the different investigated inhibitors

\begin{tabular}{|l|l|l|l|}
\hline Treatment & Transporter & $\begin{array}{l}\text { OFLX uptake } \\
\text { change (\%) }\end{array}$ & $\begin{array}{l}\text { LVFX uptake } \\
\text { change (\%)* }\end{array}$ \\
\hline Cyclosporine A & P-gP & 15 & 80 \\
\hline Verapamil & P-gP & 20 & 90 \\
\hline Quercetin & P-gP & 25 & 130 \\
\hline Omeprazole & BCRP & 15 & 50 \\
\hline Cimetidine & OCT & 30 & 50 \\
\hline Probenecid & OAT & -10 & -4 \\
\hline Lactic acid & MCT & -30 & 440 \\
\hline
\end{tabular}

Notes: OFLX data were calculated from the data presented herein. LVFX data were calculated from the study by Polachek et al. ${ }^{25} * P<0.05$.

Abbreviations: FQ, fluoroquinolone; LVFX, levofloxacin; MCT, monocarboxylate transporter; OAT, organic anion transporter; OCT, organic cation transporter; OFLX, ofloxacin. 
in Table 1. The $S L C 22 A$ gene superfamily encodes the 1-7 subtypes of the OATs, while cimetidine was reported to inhibit the efflux of substrates of OAT3 from renal cells. ${ }^{28,29}$ However, the only transporter member that is demonstrated to be present in the human syncytiotrophoblast is OAT4, which is positioned at the fetal side of the placental membrane. ${ }^{24}$ On the other hand, Zhou et al ${ }^{30}$ reported that OAT4 was not detected in wild-type BeWo cells. In fact, the lack of manifestation of this major influx transporter reflects one of the drawbacks of these monolayer cells to mimic the barrier functions of the placenta in pregnant humans. Thus, the main activity in the case of cimetidine must be attributed to its inhibitory effect on OCTs. While OCT3 and OCTN2 are documented to exist in the human placenta, ${ }^{24}$ only the latter member of the $S L C 22 A$ gene superfamily was reported to be functional in BeWo cells. ${ }^{31}$ The fact that cimetidine elevated the internal cellular levels of LVFX strongly suggests that this agent inhibits the efflux transport of the drug, demonstrating the bidirectional properties of OCTN2 to transport molecules in and out of the tissues. Indeed, carnitine, the major substrate of OCTN2, experiences efflux and influx that may change in relation to $\mathrm{pH}$ or the presence of $\mathrm{Na}^{+} .{ }^{23}$ In addition, when the inhibitory effects of OFLX and LVFX were assessed on the internalization of carnitine into BeWo cultures, the FQs exhibited a significant reduction in the uptake of this OCTN2 substrate (35\% and $65 \%$, respectively). Moreover, LVFX was confirmed to perform as an inhibitor of OCTN2 rather than a substrate, since the transport of this FQ is independent of sodium ions and since carnitine lacks inhibition properties on its transport. ${ }^{32}$

Another bidirectional transporter family is the membranebound MCT that mostly carry lactate and ketone bodies. Among its members, MCT1, MCT4-6, MCT8, and MCT10 are the subtypes that were found in the human placenta. ${ }^{33}$ In previous publications, the influx toward the tissue outweighed the apical cellular efflux. ${ }^{21}$ Consequently, the overall tendency observed in Figure 7 demonstrated a reduction of $30 \%$ in the OFLX uptake into BeWo cells pre-incubated with lactic acid, although this effect was non-significant. Actually, this competitive inhibition was also demonstrated when the uptake of acetic acid to BeWo monolayers was investigated in the presence of benzoic acid and vice versa. ${ }^{34}$

Surprisingly, the LVFX levels were considerably elevated when this carboxylic acid was evaluated in similar conditions (Table 1). Accordingly, these data may imply that MCTs in BeWo cells regulate differently the transport of these two FQs: while LVFX is transported out of the cells, OFLX is carried intracellularly. Furthermore, quercetin is also recognized for inhibiting MCTs in addition to P-gp..$^{23,35}$ Table 1 shows that this agent caused a noticeable increase in the cellular levels of LVFX attributed to both transporters in the BeWo cells.

Overall, our data indicate that the D enantiomer of the racemic mixture was not subjected to major influx and efflux transport, while multiple transporters are involved in the transfer of the levorotatory enantiomer in and out of the brush-border placental barrier.

These findings indicate that OFLX inflows into BeWo cells by means of a non-saturable mechanism, while the penetration of its biologically active optical isomer is regulated by active cellular internalization. These conclusions are further supported by the data presented in Figure 2. Polachek et al ${ }^{25}$ also demonstrated a biphasic cellular internalization of LVFX into BeWo monolayers carried out by various transporters followed by a nonsaturable process. In addition, a similar uptake pattern to LVFX was reported by Utoguchi et al, in which benzoic acid exhibited initial rapid transporter-mediated uptake into BeWo cells. This saturable course of uptake was followed by a non-saturable passage that was demonstrated at higher concentrations of the substrate. ${ }^{34}$

In a large population-based study, Daud et al inspected congenital anomalies among children of mothers who administered drug substrates of placental transporters during pregnancies. While OFLX is a substrate of BCRP in humans, the authors revealed a non-significant relationship between this FQ and congenital anomalies. In fact, in view of the following transporter proteins: OCT3, OAT4, MRP1, OATP2B1, ENT1, BCRP, MCT1, MCT4, MCT8, and MCT10, all the 58 drugs that were investigated were not related to the risk of congenital anomalies. These conclusions were also confirmed for valproic acid that was the substrate of three transporters: OAT4, MCT1, and MCT4. ${ }^{22}$ Nonetheless, in vitro experiments on BeWo cells showed substantial biphasic uptake composed of saturable and non-saturable segments. The internalization mechanism of valproic acid was essentially governed by active carrier-mediated process. ${ }^{20}$

Although the data presented above may suggest a passive diffusion mechanism of OFLX to BeWo monolayers, our previous ex vivo findings showed that the transplacental transport of OFLX was rather low, indicating that this FQ may not passively cross to the fetal compartment. In this study, using the perfusion method of human term placentae, the transplacental transport percentages of OFLX compared to a passively transported probe (antipyrine) were 3.7 and 13.4 , respectively. ${ }^{36}$ 
Although the entire set of agents that was investigated showed non-significant effects on the apical cellular uptake of OFLX, Figure 1 reveals a noticeable reduction in the normalized concentration of this FQ over time, following the 10-minute peak. This fact may indicate that OFLX is subjected to efflux transport occurring after extended time intervals. However, the efflux transporters that were evaluated in this study (P-gp and BCRP) failed to support this proposed mechanism, suggesting that other transporters may be involved in the transport of this drug. One of the major transporter proteins expressed in human placental barrier is multidrug resistance-associated proteins (MRPs). Their subtypes MRP1, MRP2, MRP3, and MRP5 are localized in the basolateral and apical sides of the syncytiotrophoblast and shield the tissue from toxic chemical substances administered to the mother. ${ }^{37}$ While the messenger RNA molecules of MRP1 and MRP2 were found in BeWo cultures, only the protein of MRP1 was detected in those cells. ${ }^{35,38-40}$ MRP1 present in the in vitro model used in this study may account for the decrease in the concentration of OFLX presented in Figure 1. The existence of this transporter and others could also account for low steady-state concentrations results of OFLX obtained in the fetus compartment using the ex vivo human placentae model. ${ }^{36}$

In light of the findings obtained in this article and the information evidenced in the literature, it seems that while the biologically active enantiomer LVFX exhibits an adequate in vitro ex vivo correlation, ${ }^{25,36}$ the transport of the racemic mixture OFLX is more complex, with indirect association between the BeWo trophoblastic cell line and the perfusion method. Therefore, careful data interpretation should be employed when examining the clinical implications of drugs administered to pregnant women, in view of studies carried out in choriocarcinoma cell lines.

\section{Disclosure}

The authors report no conflicts of interest in this work.

\section{References}

1. de Sarro A, de Sarro G. Adverse reactions to fluoroquinolones. an overview on mechanistic aspects. Curr Med Chem. 2001;8(4):371-384.

2. Kocsis B, Szabo D. New treatment options for lower respiratory tract infections. Expert Opin Pharmacother. 2017;18(13):1345-1355.

3. Paul T, Liu J, Machesky ML, Strathmann TJ. Adsorption of zwitterionic fluoroquinolone antibacterials to goethite: a charge distribution-multisite complexation model. J Colloid Interface Sci. 2014;428:63-72.

4. Candel FJ, Peñuelas M. Delafloxacin: design, development and potential place in therapy. Drug Des Devel Ther. 2017;11:881-891.

5. Drlica K, Zhao X. DNA gyrase, topoisomerase IV, and the 4-quinolones. Microbiol Mol Biol Rev. 1997;61(3):377-392.

6. Wolfson JS, Hooper DC. Fluoroquinolone antimicrobial agents. Clin Microbiol Rev. 1989;2(4):378-424.
7. Loebstein R, Addis A, Ho E, et al. Pregnancy outcome following gestational exposure to fluoroquinolones: a multicenter prospective controlled study. Antimicrob Agents Chemother. 1998;42(6):1336-1339.

8. Bookstaver PB, Bland CM, Griffin B, Stover KR, Eiland LS, Mclaughlin M. A Review of Antibiotic Use in Pregnancy. Pharmacotherapy. 2015;35(11):1052-1062.

9. Peled Y, Friedman S, Hod M, Merlob P. Ofloxacin during the second trimester of pregnancy. DICP. 1991;25(11):1181-1182.

10. Hui P. Molecular diagnosis of gestational trophoblastic disease. Expert Rev Mol Diagn. 2010;10(8):1023-1034.

11. Hui P. Gestational Trophoblastic Disease: Diagnostic and Molecular Genetic Pathology. New York: Humana Press; 2011.

12. Yabe S, Alexenko AP, Amita M, et al. Comparison of syncytiotrophoblast generated from human embryonic stem cells and from term placentas. Proc Natl Acad Sci U S A. 2016;113(19):E2598-E2607.

13. Orendi K, Kivity V, Sammar M, et al. Placental and trophoblastic in vitro models to study preventive and therapeutic agents for preeclampsia. Placenta. 2011;32(Suppl):S49-S54.

14. Bode CJ, Jin H, Rytting E, Silverstein PS, Young AM, Audus KL. In vitro models for studying trophoblast transcellular transport. Methods Mol Med. 2006;122:225-239.

15. Li H, van Ravenzwaay B, Rietjens IM, Louisse J. Assessment of an in vitro transport model using BeWo b30 cells to predict placental transfer of compounds. Arch Toxicol. 2013;87(9):1661-1669.

16. Guo J, Song D, Han F, et al. In vitro transport mechanism of psoralen in a human placental cell line (BeWo cells). Planta Med. 2015;81(2): $138-144$.

17. Pascolo L, Fernetti C, Garcia-Mediavilla MV, Ostrow JD, Tiribelli C. Mechanisms for the transport of unconjugated bilirubin in human trophoblastic BeWo cells. FEBS Lett. 2001;495(1-2):94-99.

18. da Silva RJ, Gomes AO, Franco PS, et al. Enrofloxacin and toltrazuril are able to reduce Toxoplasma gondii growth in human BeWo trophoblastic cells and villous explants from human third trimester pregnancy. Front Cell Infect Microbiol. 2017;7:340.

19. Heaton SJ, Eady JJ, Parker ML, et al. The use of BeWo cells as an in vitro model for placental iron transport. Am J Physiol Cell Physiol. 2008;295(5):C1445-C1453.

20. Ushigome F, Takanaga H, Matsuo H, et al. Uptake mechanism of valproic acid in human placental choriocarcinoma cell line (BeWo). Eur J Pharmacol. 2001;417(3):169-176.

21. Utoguchi N, Audus KL. Carrier-mediated transport of valproic acid in BeWo cells, a human trophoblast cell line. Int J Pharm. 2000;195(1-2): $115-124$.

22. Daud AN, Bergman JE, Oktora MP, et al. Maternal use of drug substrates of placental transporters and the effect of transporter-mediated drug interactions on the risk of congenital anomalies. PLoS One. 2017; 12(3):e0173530.

23. Vähäkangas K, Myllynen P. Drug transporters in the human bloodplacental barrier. Br J Pharmacol. 2009;158(3):665-678.

24. Roth M, Obaidat A, Hagenbuch B, Oatps HB. OATPs, OATs and OCTs: the organic anion and cation transporters of the SLCO and SLC22A gene superfamilies. Br J Pharmacol. 2012;165(5):1260-1287.

25. Polachek H, Holcberg G, Polachek J, et al. Carrier-mediated uptake of Levofloxacin by BeWo cells, a human trophoblast cell line. Arch Gynecol Obstet. 2010;281(5):833-838.

26. Lowry OH, Rosebrough NJ, Farr AL, Randall RJ. Protein measurement with the Folin phenol reagent. J Biol Chem. 1951;193(1):265-275.

27. Feinshtein V, Erez O, Ben-Zvi Z, et al. Cannabidiol changes P-gp and BCRP expression in trophoblast cell lines. PeerJ. 2013;1:e153.

28. Nagata Y, Kusuhara H, Hirono S, Endou H, Sugiyama Y. Carriermediated uptake of H2-receptor antagonists by the rat choroid plexus: involvement of rat organic anion transporter 3. Drug Metab Dispos. 2004;32(9):1040-1047.

29. Tahara H, Kusuhara H, Endou H, et al. A species difference in the transport activities of $\mathrm{H} 2$ receptor antagonists by rat and human renal organic anion and cation transporters. J Pharmacol Exp Ther. 2005; 315(1):337-345. 
30. Zhou F, Illsley NP, You G. Functional characterization of a human organic anion transporter hOAT4 in placental BeWo cells. Eur $J$ Pharm Sci. 2006;27(5):518-523.

31. Chang TT, Shyu MK, Huang MC, et al. Hypoxia-mediated downregulation of OCTN2 and PPAR $\alpha$ expression in human placentas and in BeWo cells. Mol Pharm. 2011;8(1):117-125.

32. Hirano T, Yasuda S, Osaka Y, et al. The inhibitory effects of fluoroquinolones on L-carnitine transport in placental cell line BeWo. Int J Pharm. 2008;351(1-2):113-118.

33. Iwanaga T, Kishimoto A. Cellular distributions of monocarboxylate transporters: a review. Biomed Res. 2015;36(5):279-301.

34. Utoguchi N, Magnusson M, Audus KL. Carrier-mediated transport of monocarboxylic acids in BeWo cell monolayers as a model of the human trophoblast. J Pharm Sci. 1999;88(12):1288-1292.

35. Myllynen P, Kummu M, Kangas T, et al. ABCG2/BCRP decreases the transfer of a food-born chemical carcinogen, 2-amino-1-methyl-6phenylimidazo[4,5-b]pyridine (PhIP) in perfused term human placenta. Toxicol Appl Pharmacol. 2008;232(2):210-217.
36. Polachek H, Holcberg G, Sapir G, et al. Transfer of ciprofloxacin, ofloxacin and levofloxacin across the perfused human placenta in vitro. Eur J Obstet Gynecol Reprod Biol. 2005;122(1):61-65.

37. Bodó A, Bakos E, Szeri F, Váradi A, Sarkadi B. The role of multidrug transporters in drug availability, metabolism and toxicity. Toxicol Lett. 2003;140-141:133133-133143.

38. Evseenko DA, Paxton JW, Keelan JA. ABC drug transporter expression and functional activity in trophoblast-like cell lines and differentiating primary trophoblast. Am J Physiol Regul Integr Comp Physiol. 2006; 290(5):R1357-R1365.

39. Mao Q. BCRP/ABCG2 in the placenta: expression, function and regulation. Pharm Res. 2008;25(6):1244-1255.

40. Serrano MA, Macias RI, Briz O, et al. Expression in human trophoblast and choriocarcinoma cell lines, BeWo, Jeg-3 and JAr of genes involved in the hepatobiliary-like excretory function of the placenta. Placenta. 2007;28(2-3):107-117.

\section{Publish your work in this journal}

Drug Design, Development and Therapy is an international, peerreviewed open-access journal that spans the spectrum of drug design and development through to clinical applications. Clinical outcomes, patient safety, and programs for the development and effective, safe, and sustained use of medicines are the features of the journal, which has also been accepted for indexing on PubMed Central. The manuscript management system is completely online and includes a very quick and fair peer-review system, which is all easy to use. Visit http://www.dovepress.com/testimonials.php to read real quotes from published authors.

Submit your manuscript here: http://www.dovepress.com/drug-design-development-and-therapy-journal 